\title{
Mammogram Segmentation using a Improved Nonlinear Access of Level Set Method
}

\author{
Saikumar Tara
}

\begin{abstract}
Image segmentation plays a serious role in field of medical image processing, forensic sciences and many more.A nonlinear approach for image segmentation leads to a critical issues compare to a linear approach. In this paper a nonlinear approach algorithm is proposed for segmentation purpose by using Bayesian rules of probability weighted force function for retrieve weak sense boundaries. This proposed method reduces the boundary leakages and also provide true boundaries of an images. An experimental setup results for an improved proposed method of snake or Level set method on mammograms gives a better results.
\end{abstract}

Key words: Bayesian rule, Image Segmentation, PDE, Contour.

\section{INTRODUCTION}

A Popular techniques used in Image segmentation are Contours or Active contours or snakes [1]. In broad spectrum snakes initiate from a curve which is closed and force the evolving curve based on Partial Differential Equation (PDE).This Partial Differential Equation is an originated from a family of Jacobi equations and obtained from Euler Lagrange's equations which is a pre-defined energy functional. The main function involved in snake model to handle graphical changing of closed curve. LSM is broadly divided into edge and region based segmentations, and contours are widely used for segmentation of images [2].

Handling on real boundaries, boundary leakages problems are complex issues with nonlinear approach. Level Set approach involves a concept of new edge indication function, force stopping approach evolve on regions with it energy based functional [5]. A novel approach for image segmentation to leads such as force functional direction on the curve evolution by non linear, these approach speed up to remove an occurrences of boundary leakages for boundaries which are weak.

\section{LEVEL SET METHOD}

The set function based on Level defines its value to zero level if an only if, it is not a true sign distance metric function with which an image will not be true. The modified level set function (LSF) is iteratively solving a PDE in the evaluation process stage of level set.

This re-modification of a level set function always executed each and every iteration for reduces the cost function. Energy functional can be define as [2]

Revised Manuscript Received on September 14, 2019.

Saikumar Tara, Senior MIEEE, Associate Professor, Department of Electronics and Communication Engineering, CMR Technical Campus (A), Hyderabad, Tamilnadu, India.(Email: tarasaikumar.ece@cmrtc.ac.in)

$$
E_{g}=|\nabla \phi| \cdot g\left[\frac{\mu}{g} \int_{\zeta} \frac{1}{2}\left(|\nabla \phi|+\frac{1}{|\nabla \phi|}-2\right) d x d y+\lambda \int_{\xi} \delta(\phi) d x d y+v \int_{\xi} H(-\phi) d x d y\right]-----(1)
$$

Where indicates domain of image and is level set function. The individual elements in equation (1) are an evolution process, weight based length curve [3]. The physical parameter in the equation (1) controls of convergence speed, ' ' is an edge indicator function can be defined as

$$
g=\frac{1}{1+\left|\nabla G_{\sigma} . I\right|^{2}}----(2)
$$

Where $G_{\sigma}$ is a Gaussian..

The external energy functional can be redefined as

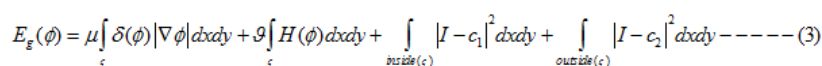

Where and both are mean average value of grayscale images $C_{1}$ for $C_{2}$ both in and out sides of the curve.

\section{IMPROVED VERSION OF NON LINEAR LEVEL SET METHODS}

The whole image consider in to two sub domain such as where and represent significantly the image and background portion of an image[6].

$$
\psi(x, y)=p\left(\frac{\Omega_{1}}{I_{1}(x, y)}\right)-t_{p}----(4)
$$

where $p\left(\frac{\Omega_{1}}{I_{1}(x, y)}\right)$ is a probability that gives the pels and tp denotes threshold .

The modified energy functional for a LSF which defined as that can be nucleus formula of the LSF for an input image and incorporate different gradients for each images. The model energy functional based on [6] which is

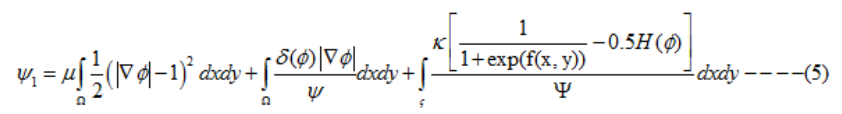

$$
\Psi=1+\left.p\left(\zeta_{1} / I^{*}\right) p\left(\frac{\zeta_{2}}{I^{*}}\right)\left|\nabla G_{\sigma}\right|^{*} I^{*}\right|^{m+1}-----(6)
$$

Published By: 
The evolution equation of the LSF is a part of PDE on which directly driving the evolve of boundaries of the curve for a partial corresponding energy function [5] approaches to minimum.

\section{EXPERIMENTAL RESULTS}

In Experiment of a specified image is containing with an uncertain and complex outline are as shown in figures [1-3].

The proposed method achieves better result with a pretty smooth closed curve because of the self-adjusted adaptive direction, nonlinear approach and weight stopping force.

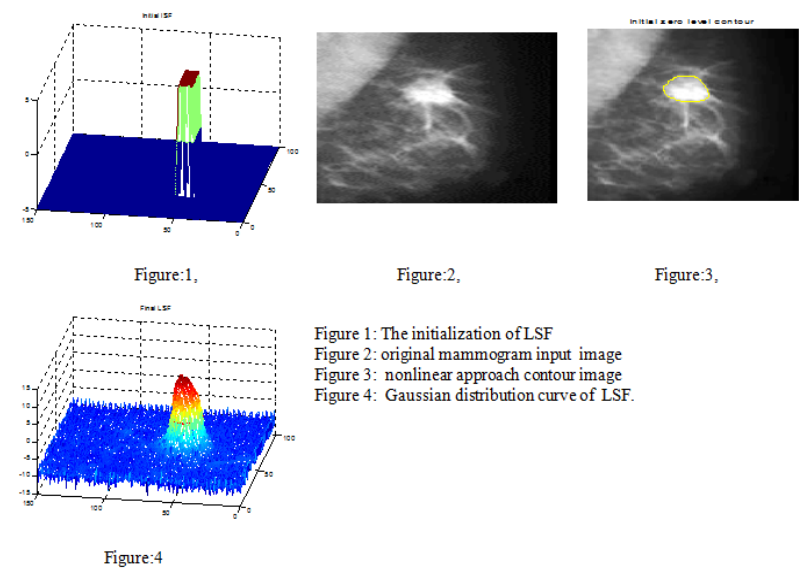

\section{CONCLUSION AND DISCUSSION}

In this paper a novel construct an improved new nonlinear probability weighted stopping force for both local and global features values and also resolves the issues which are encounter in early stages about shrink and expand of contour curve. A non-linearly approach can be avoid leakages in the boundaries and weight stopped function of false boundaries involved with noise.

Finally combustion of Bayesian rule for non linear LSM gives better results even it is applicable for multiple level set functions.

\section{REFERENCES}

1. Y. Du, E. Arslanturk, Z. Zhou, and C. Belcher, "Videobased non cooperative iris image segmentation," IEEE Trans. Syst., Man, Cybern. B,Cybern., vol. 41, no. 1, pp. 64-74, Feb. 2011.

2. C. Li, C. Xu, C. Gui, and M. D. Fox, "Level set evolution without re-initialization: A new variational formulation," in Proc. IEEE Conf. Computer Vision Pattern Recog., 2005, pp. 430-436.

3. V. Caselles, R. Kimmel, and G. Sapiro, "Geodesic active contours," Int. J. Computer Vision vol. 22, no. 1, pp. 6179, Feb. 1997.

4. T. F. Chan and L. A. Vese, "Active contours without edges," IEEE Trans. Image Process., vol. 10, no. 2, pp. 266-277, Feb. 2001.

5. Bin Wang, X. Gao, D. Tao, and X. Li, " A Non Linear Adaptive Level Set for Image Segmentation", IEEE Transaction on Cybernetics, 2014.

6. Saikumar Tara, R.Nirmala Devi, "Radon Transform based modified nonlinear access for segmentation of mammogram applications", International Journal of Recent Technology and Engineering, vol (8),Iss(2), July 2019. 